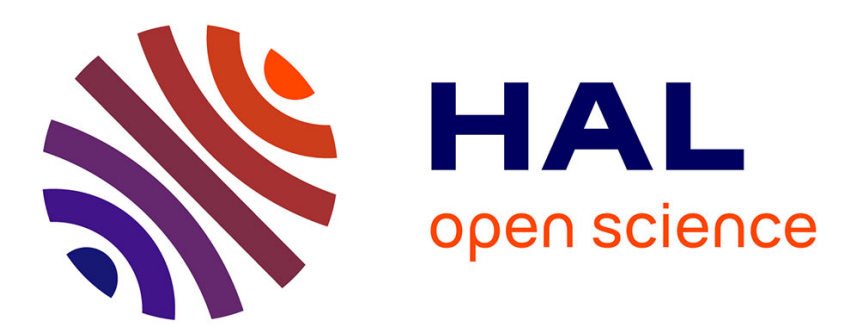

\title{
On the spectrum and stiffness of an elastic body with surface stresses
}

\author{
Holm Altenbach, Victor A. Eremeyev, Leonid P. Lebedev
}

\section{To cite this version:}

Holm Altenbach, Victor A. Eremeyev, Leonid P. Lebedev. On the spectrum and stiffness of an elastic body with surface stresses. Journal of Applied Mathematics and Mechanics / Zeitschrift für Angewandte Mathematik und Mechanik, 2011, 91 (9), pp.699-710. hal-00824109

\section{HAL Id: hal-00824109 \\ https://hal.science/hal-00824109}

Submitted on 21 May 2013

HAL is a multi-disciplinary open access archive for the deposit and dissemination of scientific research documents, whether they are published or not. The documents may come from teaching and research institutions in France or abroad, or from public or private research centers.
L'archive ouverte pluridisciplinaire HAL, est destinée au dépôt et à la diffusion de documents scientifiques de niveau recherche, publiés ou non, émanant des établissements d'enseignement et de recherche français ou étrangers, des laboratoires publics ou privés. 


\title{
On the spectrum and stiffness of an elastic body with surface stresses
}

\author{
Holm Altenbach ${ }^{1, *}$, Victor A. Eremeyev ${ }^{1,2, * *}$, and Leonid P. Lebedev ${ }^{3, * * *}$ \\ ${ }^{1}$ Lehrstuhl für Technische Mechanik, Zentrum für Ingenieurwissenschaften, Martin-Luther-Universität Halle-Wittenberg, \\ 06099 Halle (Saale), Germany \\ 2 South Scientific Center of RASci \& South Federal University, Milchakova St. 8a, 344090 Rostov on Don, Russia \\ ${ }^{3}$ Universidad Nacional de Colombia, Cr. 45, \# 26-85, Bogotá D.C., Colombia
}

\begin{abstract}
Key words Surface stresses, eigenfrequencies, energy spaces of Sobolev's type, Rayleigh variational principle, Courant's maximum-minimum principle.

A mathematical investigation of the eigenvalue problems for elastic bodies including surface stresses is presented. Weak setup of the problems is based on the Rayleigh variational principle. Certain spectral properties are established for the problems under consideration. In particular, bounds for the eigenfrequencies of an elastic body with surface stresses are presented. These bounds demonstrate increases in both the rigidity of the body and of the eigenfrequencies over those of the body with surface stresses neglected.
\end{abstract}

\section{Introduction}

The development of nanomechanics has generated interest in the theory of elasticity with surface stresses [6]. Surface effects can explain the abnormal properties of nanomaterials. In particular, it has been shown [7] that by taking into account surface elasticity, we obtain the stiffness increase of nanoporous materials in comparison with their usual models. In certain proposed models for surface stresses $[10,12,22,25,26]$, the influence of surface elasticity on the natural vibrations of microand nano-sized beams is analysed. The theory of elasticity with surface stresses can be found in, e.g., [11, 18, 19, 23]. In this theory, an elastic body with surface stresses is considered as a linear elastic body having an elastic membrane glued to all or part of its surface. Existence of a weak solution and related problems in linear elasticity with surface stresses are investigated in [2]. Existence of classical solutions to boundary value problems with surface reinforcements is established in $[20,21]$.

In this paper we show that the ordered eigenfrequencies of an elastic body with a non-fixed boundary portion can only increase if we consider the action of surface stresses on this portion. That is, for a bounded elastic body with a fixed boundary portion and surface stresses acting on the rest of the boundary, the ordered eigenfrequencies are greater than or equal to the corresponding eigenfrequencies of the body when surface stresses are neglected. Surface elasticity does not affect the density of the body; this means that by adding the action of surface stresses, we increase the stiffness of the body. Moreover, we prove that the ordered eigenfrequencies of an elastic body with surface stresses can only increase if we fix the whole boundary. Hence we obtain lower and upper bounds for the eigenfrequencies of an elastic body with surface stresses.

\section{Basic relations of linear elasticity with surface stresses}

Suppose an elastic body occupies a bounded volume $V \subset \mathbb{R}^{3}$ with a piecewise smooth boundary $\Omega$. We consider a problem with mixed boundary conditions. Let a nonempty portion $\Omega_{1}$ of $\Omega$ be fixed so that the displacement $\boldsymbol{u}=\boldsymbol{0}$ at each point of $\Omega_{1}$. Surface stresses $\tau$ act over the remainder of the boundary $\Omega_{2}=\Omega \backslash \Omega_{1}$. The boundary value problem is given by the relations $[6,7,11,18]$

$$
\begin{aligned}
& \nabla \cdot \boldsymbol{\sigma}=\rho \ddot{\boldsymbol{u}}, \quad \boldsymbol{x} \in V, \\
& \left.\boldsymbol{u}\right|_{\Omega_{1}}=\mathbf{0},\left.\quad \boldsymbol{n} \cdot \boldsymbol{\sigma}\right|_{\Omega_{2}}=\nabla_{S} \cdot \boldsymbol{\tau}, \quad \boldsymbol{x} \in \Omega,
\end{aligned}
$$

* $\quad$ E-mail: holm.altenbach@iw.uni-halle.de

** Corresponding author E-mail: eremeyev.victor@gmail.com; victor.eremeyev@iw.uni-halle.de

*** E-mail: llebedev@unal.edu.co 
where $\boldsymbol{\sigma}$ is the stress tensor, $\nabla$ is the three-dimensional nabla operator, $\rho$ is the material density, $\boldsymbol{n}$ is the exterior unit normal to $\Omega, \boldsymbol{\tau}$ is the surface stress tensor, $\nabla_{S}=\nabla-\boldsymbol{n} \partial / \partial z$ is the surface nabla operator, and $z$ is the coordinate along $\boldsymbol{n}$. An overdot denotes differentiation with respect to time $t$. The dot symbol "." between variables stands for the dot product in $\mathbb{R}^{3}$. For simplicity, we consider a homogeneous boundary value problem without volume forces or external surface forces.

In the case of an isotropic material, Eqs. (1)-(2) should be supplemented as follows. Inside $V$ we require

$$
\begin{aligned}
& W=W(\varepsilon) \equiv \frac{1}{2} \lambda \operatorname{tr}^{2} \varepsilon+\mu \varepsilon: \varepsilon, \\
& \boldsymbol{\sigma}=\frac{\partial W}{\partial \varepsilon} \equiv 2 \mu \varepsilon+\lambda \mathbf{I} \operatorname{tr} \varepsilon, \quad \varepsilon=\varepsilon(\boldsymbol{u}) \equiv \frac{1}{2}\left(\nabla \boldsymbol{u}+(\nabla \boldsymbol{u})^{T}\right),
\end{aligned}
$$

where $W$ is the volume energy density, $\varepsilon$ is the volume strain tensor, $\lambda, \mu$ are Lamé's moduli for the bulk material, and the symbol ":" stands for the inner product in the space of second order tensors. Because the displacement vector for points of $\Omega$ is the continuation of $\boldsymbol{u}$ in the volume, it is also denoted by $\boldsymbol{u}$. On $\Omega_{2}$ we require

$$
\begin{aligned}
& U=U(\boldsymbol{\epsilon}) \equiv \frac{1}{2} \lambda_{S} \operatorname{tr}^{2} \boldsymbol{\epsilon}+\mu_{S} \boldsymbol{\epsilon}: \boldsymbol{\epsilon}, \\
& \boldsymbol{\tau}=\frac{\partial U}{\partial \boldsymbol{\varepsilon}} \equiv 2 \mu_{S} \boldsymbol{\epsilon}+\lambda_{S} \mathbf{A} \operatorname{tr} \boldsymbol{\epsilon}, \quad \boldsymbol{\epsilon}=\boldsymbol{\epsilon}(\boldsymbol{u}) \equiv \frac{1}{2}\left(\nabla_{S} \boldsymbol{u} \cdot \mathbf{A}+\mathbf{A} \cdot\left(\nabla_{S} \boldsymbol{u}\right)^{T}\right),
\end{aligned}
$$

where $U$ is the energy density of the surface deformation, $\boldsymbol{\epsilon}$ is the surface strain tensor, $\mathbf{A}=\mathbf{I}-\boldsymbol{n} \otimes \boldsymbol{n}$ is the surface unit tensor, $\mathbf{I}$ is the three-dimensional unit tensor, and $\lambda_{S}, \mu_{S}$ are the surface elastic Lamé's moduli.

We assume that $W$ and $U$ are positive definite quadratic forms with respect to their arguments:

$$
W(\varepsilon) \geq c_{1} \varepsilon: \varepsilon, \quad U(\epsilon) \geq c_{2} \epsilon: \epsilon,
$$

where $c_{1}, c_{2}$ are positive constants. From (7) it follows that

$$
3 \lambda+2 \mu>0, \quad \mu>0, \quad \lambda_{S}+\mu_{S}>0, \quad \mu_{S}>0 .
$$

When $\lambda_{S}=\mu_{S}=0$, Eqs. (1)-(2) reduce to the mixed boundary value problem of classical linear elasticity:

$$
\nabla \cdot \boldsymbol{\sigma}=\rho \ddot{\boldsymbol{u}}, \quad \boldsymbol{x} \in V ;\left.\quad \boldsymbol{u}\right|_{\Omega_{1}}=\mathbf{0},\left.\quad \boldsymbol{n} \cdot \boldsymbol{\sigma}\right|_{\Omega_{2}}=\mathbf{0} .
$$

When $\lambda_{S}, \mu_{S} \rightarrow \infty$, because $\boldsymbol{u}=\mathbf{0}$ on the boundary of $\Omega_{2}$, Eqs. (1)-(2) reduce to the dynamic equations of classical elasticity for a body with fixed boundary:

$$
\nabla \cdot \boldsymbol{\sigma}=\rho \ddot{\boldsymbol{u}}, \quad \boldsymbol{x} \in V ;\left.\quad \boldsymbol{u}\right|_{\Omega}=\mathbf{0} .
$$

These relations for the problems allow us to compare their ordered sets of eigenfrequencies.

\section{Eigenoscillations}

Let us seek the displacement field in the form $\boldsymbol{u}(\boldsymbol{x}, t)=\boldsymbol{w}(\boldsymbol{x}) \exp (i \omega t)$. Eqs. (1)-(2) reduce to

$$
\nabla \cdot \boldsymbol{\sigma}=-\rho \omega^{2} \boldsymbol{w}, \quad \boldsymbol{x} \in V ;\left.\quad \boldsymbol{w}\right|_{\Omega_{1}}=\mathbf{0},\left.\quad\left(\boldsymbol{n} \cdot \boldsymbol{\sigma}-\nabla_{S} \cdot \boldsymbol{\tau}\right)\right|_{\Omega_{2}}=\mathbf{0} .
$$

These, when supplemented with the appropriately transformed relations (3)-(6), constitute the eigenfrequency problem for a body $V$ with surface stresses. We refer to it as Problem $P_{s s}$.

The spectrum of Problem $P_{s s}$ will be compared with the spectra of two problems obtained by substituting $\boldsymbol{u}(\boldsymbol{x}, t)=$ $\boldsymbol{w}(\boldsymbol{x}) \exp (i \omega t)$ into (8) and (9). For (8), the eigenoscillation equations are

$$
\nabla \cdot \boldsymbol{\sigma}=-\rho \omega^{2} \boldsymbol{w}, \quad \boldsymbol{x} \in V ;\left.\quad \boldsymbol{w}\right|_{\Omega_{1}}=\mathbf{0},\left.\quad \boldsymbol{n} \cdot \boldsymbol{\sigma}\right|_{\Omega_{2}}=\mathbf{0} .
$$

Supplemented with the correspondingly transformed relations (4), they constitute Problem $P_{f}$. Finally, the equations

$$
\nabla \cdot \boldsymbol{\sigma}=-\rho \omega^{2} \boldsymbol{w}, \quad \boldsymbol{x} \in V ;\left.\quad \boldsymbol{w}\right|_{\Omega}=\mathbf{0}
$$

supplemented with the correspondingly transformed relations (4), constitute eigenfrequency Problem $P_{0}$ for a body $V$ with fixed boundary. 
Note that the operators of the boundary value problems for elastic bodies with surface stresses have properties similar to those for the operators of linear elasticity; these are well established, cf. for example $[4,9,15]$. Weak setup of boundary value problems in the theory of elasticity with surface stresses is studied in [2]. We present the necessary definitions.

For Problem $P_{s s}$, we introduce the energy space $\boldsymbol{E}$ with the inner product

$$
\langle\boldsymbol{u}, \boldsymbol{v}\rangle_{\boldsymbol{E}}=\int_{V}[\lambda \operatorname{tr} \boldsymbol{\varepsilon}(\boldsymbol{u}) \operatorname{tr} \boldsymbol{\varepsilon}(\boldsymbol{v})+2 \mu \varepsilon(\boldsymbol{u}): \varepsilon(\boldsymbol{v})] d V+\int_{\Omega_{2}}\left[\lambda_{S} \operatorname{tr} \boldsymbol{\epsilon}(\boldsymbol{u}) \operatorname{tr} \boldsymbol{\epsilon}(\boldsymbol{v})+2 \mu_{S} \boldsymbol{\epsilon}(\boldsymbol{u}): \boldsymbol{\epsilon}(\boldsymbol{v})\right] d \Omega
$$

and the norm

$$
\|\boldsymbol{u}\|_{\boldsymbol{E}}^{2}=\langle\boldsymbol{u}, \boldsymbol{u}\rangle_{\boldsymbol{E}}
$$

Roughly speaking, an element $\boldsymbol{u} \in \boldsymbol{E}$ belongs to both $\left(W^{1,2}(V)\right)^{3}$ and $\left(W^{1,2}\left(\Omega_{2}\right)\right)^{3}$.

An eigenpair $\left(\omega_{k}, \boldsymbol{w}_{k}\right)$, where $\boldsymbol{w}_{k} \neq \mathbf{0}$ and $\omega_{k}$ is an eigenfrequency, satisfies

$$
\left\langle\boldsymbol{w}_{k}, \boldsymbol{v}\right\rangle_{\boldsymbol{E}}=\omega_{k}^{2} \int_{V} \rho \boldsymbol{w}_{k} \cdot \boldsymbol{v} d V
$$

for any $\boldsymbol{v} \in \boldsymbol{E}$. It has been shown [2] that the set of eigenfrequencies $\left\{\omega_{k}\right\}, 0<\omega_{\min }=\omega_{1} \leq \omega_{2} \leq \omega_{3}, \ldots$, of Problem $P_{s s}$ is countable, that its only point of accumulation is infinite, and that to each $\omega_{k}$ there corresponds an eigenmode $\boldsymbol{w}_{k}$ which can be selected so that

$$
\int_{V} \rho \boldsymbol{w}_{k} \cdot \boldsymbol{w}_{m} d V=\delta_{k m}, \quad \text { and }\left\langle\boldsymbol{w}_{i}, \boldsymbol{w}_{j}\right\rangle_{\boldsymbol{E}}=0 \text { for } i \neq j,
$$

where $\delta_{k m}$ is Kronecker's symbol. Moreover, the set of $\boldsymbol{w}_{k}, k=1,2, \ldots$, is complete in $\left(L^{2}(V)\right)^{3}$ and $\boldsymbol{E}$.

For Problems $P_{f}$ and $P_{0}$, the results for the eigensolutions are similar to those stated above; cf. [14, 15] among others. For Problem $P_{f}$, the energy space $\boldsymbol{H}$ is the completion of the set of vector functions $\boldsymbol{u} \in\left(C^{(2)}(\bar{V})\right)^{3}$ that vanish on $\Omega_{1}$. The norm $\|\cdot\|_{\boldsymbol{H}}$ is induced by the inner product

$$
\langle\boldsymbol{u}, \boldsymbol{v}\rangle_{\boldsymbol{H}}=\int_{V}[\lambda \operatorname{tr} \varepsilon(\boldsymbol{u}) \operatorname{tr} \varepsilon(\boldsymbol{v})+2 \mu \varepsilon(\boldsymbol{u}): \varepsilon(\boldsymbol{v})] d V
$$

To enable the use of Sobolev's embedding theorems [1], we suppose $V$ has a piecewise smooth boundary that satisfies the cone condition. The boundary of $\Omega_{2}$ should be piecewise smooth and should also satisfy the cone condition on the plane of its inner coordinates.

The weak setup of the eigenvalue Problem $P_{f}$ is given by

$$
\left\langle\boldsymbol{w}_{k}, \boldsymbol{v}\right\rangle_{\boldsymbol{H}}=\left(\omega_{k}^{f}\right)^{2} \int_{V} \rho \boldsymbol{w}_{k} \cdot \boldsymbol{v} d V
$$

which holds for all $\boldsymbol{v} \in \boldsymbol{H}$. Here $\omega_{k}^{f}$ is an eigenfrequency. We retain the notation $\boldsymbol{w}_{k}$ for eigenmodes, although these are in general distinct for different problems. To formulate the results for Problem $P_{f}$ from those presented above for Problem $P_{s s}$, we change the space $\boldsymbol{E}$ to $\boldsymbol{H}$ and the inner product to (15). The set of ordered eigenmodes of Problem $P_{f}$ is complete in $\boldsymbol{H}$ and $\left(L^{2}(V)\right)^{3}$; furthermore,

$$
\int_{V} \rho \boldsymbol{w}_{k} \cdot \boldsymbol{w}_{m} d V=\delta_{k m} \quad \text { and } \quad\left\langle\boldsymbol{w}_{i}, \boldsymbol{w}_{j}\right\rangle_{\boldsymbol{H}}=0 \quad \text { for } \quad i \neq j .
$$

A particular case of Problem $P_{f}$ is Problem $P_{0}$. Here the elements $\boldsymbol{w}$ vanish on $\Omega$. The energy space $\boldsymbol{H}_{0}$, a subspace of $\boldsymbol{H}$, is the completion of the set of vector functions $\boldsymbol{u} \in\left(C_{0}^{(2)}(\bar{V})\right)^{3} \cdot \boldsymbol{H}_{0}$ inherits the norm $\|\cdot\|_{\boldsymbol{H}}$ of $\boldsymbol{H}$. The equation for the eigensolutions coincides with (16), which must hold for all $\boldsymbol{v} \in \boldsymbol{H}_{0}$. The spectral properties are the same as those of Problem $P_{f}$, including the relations (17).

We recall that $\boldsymbol{u} \in \boldsymbol{H}$ means that $\boldsymbol{u} \in\left(W^{1,2}(V)\right)^{3}$ and, by the trace theorem [1], $\boldsymbol{u} \in\left(W^{1 / 2,2}\left(\Omega_{2}\right)\right)^{3}$. The relation $\boldsymbol{u} \in \boldsymbol{H}_{0}$ means that $\boldsymbol{u} \in\left(W_{0}^{1,2}(V)\right)^{3}$. We may consider $\boldsymbol{E}$ as a subset of $\boldsymbol{H}$, and $\boldsymbol{H}_{0}$ as a subset of $\boldsymbol{E}$. We will use this in what follows. 


\section{Rayleigh quotient and some properties of the spectrum}

\subsection{The least eigenfrequency}

The estimation of the least eigenfrequency is an important problem from an engineering standpoint. For solids with surface stresses, the eigenvalue Problem $P_{s s}$ can be formulated using Rayleigh's variational principle [2].

Let $R(\boldsymbol{w})$ be Rayleigh's quotient for the body with surface stresses:

$$
R(\boldsymbol{w})=\frac{\|\boldsymbol{w}\|_{\boldsymbol{E}}^{2}}{K(\boldsymbol{w})}, \quad K(\boldsymbol{w})=\int_{V} \rho \boldsymbol{w} \cdot \boldsymbol{w} d V .
$$

The squared least eigenfrequency $\omega_{\min }$ is determined as the infimum of $R(\boldsymbol{w})$ :

$$
\omega_{\min }^{2}=\inf _{\boldsymbol{w} \in \boldsymbol{E}} R(\boldsymbol{w})
$$

For Problems $P_{f}$ and $P_{0}$, Rayleigh's quotient is the same as in linear elasticity, i.e.,

$$
R_{0}(\boldsymbol{w})=\frac{\|\boldsymbol{w}\|_{\boldsymbol{H}}^{2}}{K(\boldsymbol{w})} .
$$

For Problem $P_{f}$, the squared least eigenfrequency $\omega_{\min }^{f}$ is the infimum of $R_{0}(\boldsymbol{w})$ over $\boldsymbol{H}$. For Problem $P_{0}$, the squared least eigenfrequency $\omega_{\min }^{\circ}$ is the infimum of $R_{0}(\boldsymbol{w})$ over $\boldsymbol{H}_{0}$.

$R(\boldsymbol{w})$ is determined on $\boldsymbol{E}$. Let us formally extend its domain to $\boldsymbol{H}$ as follows. An element of $\boldsymbol{E}$ can be considered as an element of $\boldsymbol{H}$, and so for this element we define the value of $R$ on $\boldsymbol{w} \in \boldsymbol{E}$. When $\boldsymbol{w} \in \boldsymbol{H}$ but $\boldsymbol{w}$ lacks the smoothness on $\Omega_{2}$ needed to associate it with an element of $\boldsymbol{E}$ and $\|\boldsymbol{w}\|_{\boldsymbol{E}}$ is undefined, then we formally put $\|\boldsymbol{w}\|_{\boldsymbol{E}}=+\infty$. By the positivity of the energy function, it is easy to see that $R_{0}(\boldsymbol{w}) \leq R(\boldsymbol{w})$ on $\boldsymbol{H}$, and $R_{0}(\boldsymbol{w})=R(\boldsymbol{w})$ if $\boldsymbol{w} \in \boldsymbol{H}_{0}$.

The properties of the spaces $\boldsymbol{E}, \boldsymbol{H}, \boldsymbol{H}_{0}$ and the functionals $R, R_{0}$ allow us to prove the following

Theorem 3.1. The least eigenfrequency of a bounded elastic body with surface stresses (Problem $P_{\text {ss }}$ ) is no less than the least eigenfrequency for the same body with free boundary $\Omega_{2}$ (Problem $\left.P_{f}\right)$, and it is no greater than the least eigenfrequency for the same body with fixed boundary (Problem $\left.P_{0}\right)$ :

$$
\omega_{\min }^{f} \leq \omega_{\min } \leq \omega_{\min }^{\circ}
$$

Proof. The proof follows from two inequality chains:

$$
\left(\omega_{\min }^{f}\right)^{2}=\inf _{\boldsymbol{w} \in \boldsymbol{H}} R_{0}(\boldsymbol{w}) \leq \inf _{\boldsymbol{w} \in \boldsymbol{E} \subset \boldsymbol{H}} R_{0}(\boldsymbol{w}) \leq \inf _{\boldsymbol{w} \in \boldsymbol{E}} R(\boldsymbol{w})=\left(\omega_{\min }\right)^{2},
$$

since $\boldsymbol{E}$ constitutes a subset of $\boldsymbol{H}$, and

$$
\left(\omega_{\min }\right)^{2}=\inf _{\boldsymbol{w} \in \boldsymbol{E}} R(\boldsymbol{w}) \leq \inf _{\boldsymbol{w} \in \boldsymbol{H}_{0} \subset \boldsymbol{E}} R(\boldsymbol{w})=\inf _{\boldsymbol{w} \in \boldsymbol{H}_{0}} R_{0}(\boldsymbol{w})=\left(\omega_{\min }^{\circ}\right)^{2} .
$$

Two results follow from the proof of Theorem 3.1.

Corollary 1. The equality $\omega_{\min }=\omega_{\min }^{f}$ holds if and only if $U\left(\boldsymbol{\epsilon}\left(\boldsymbol{w}_{\min }\right)\right)=0$ on $\Omega_{2}$.

By positive definiteness, $U=0$ if and only if $\boldsymbol{\epsilon}\left(\boldsymbol{w}_{\min }\right)=\mathbf{0}$ on $\Omega_{2}$. Hence the displacement $\boldsymbol{w}_{\min }$ of $\Omega_{2}$ describes an infinitesimal isometric deformation of $\Omega_{2}$. In particular, $\boldsymbol{\epsilon}=\mathbf{0}$ if $\boldsymbol{w}_{\min }$ describes a rigid body motion.

Corollary 2. The equality $\omega_{\min }=\omega_{\min }^{\circ}$ holds if and only if $\boldsymbol{w}_{\min }=\mathbf{0}$ on $\Omega_{2}$, which is when $\boldsymbol{w}_{\min } \in \boldsymbol{H}_{0}$.

Cases when $\boldsymbol{\epsilon}\left(\boldsymbol{w}_{\min }\right)=\mathbf{0}$ or $\boldsymbol{w}_{\min }=\mathbf{0}$ on $\Omega_{2}$ should be rare for an elastic body of general shape and with general boundary conditions. Hence, in general we can expect the strict inequalities

$$
\omega_{\min }^{f}<\omega_{\min }<\omega_{\min }^{\circ}
$$

This extends the inequality $\omega_{\min }^{f}<\omega_{\min }^{\circ}$, well known in the theory of elasticity and mathematical physics [5, 24].

The least eigenfrequency $\omega_{\min }$ depends on $\lambda_{S}$ and $\mu_{S}$. An increase in the surface elastic moduli implies an increase in the least eigenfrequency of Problem $P_{s s}$. Indeed, let us consider two bodies of equal shape and equal internal moduli $\lambda$ and $\mu$, but with different values of $\lambda_{S}$ and $\mu_{S}$. Denote the surface moduli of the bodies by $\lambda_{S}^{(1)}, \mu_{S}^{(1)}$ and $\lambda_{S}^{(2)}, \mu_{S}^{(2)}$, respectively. Denote the least eigenfrequencies of the bodies by $\omega_{\min }^{(1)}$ and $\omega_{\min }^{(2)}$, respectively. 
Theorem 3.2. Let

$$
0<\mu_{S}^{(1)} \leq \mu_{S}^{(2)}, \quad 0<\lambda_{S}^{(1)}+\mu_{S}^{(1)} \leq \lambda_{S}^{(2)}+\mu_{S}^{(2)} .
$$

Then

$$
\omega_{\min }^{(1)} \leq \omega_{\min }^{(2)}
$$

Proof. The proof follows immediately from the inequality $R_{(1)}(\boldsymbol{w}) \leq R_{(2)}(\boldsymbol{w})$, where $R_{(\alpha)}(\boldsymbol{w}), \alpha=1,2$, are Rayleigh's quotients for the bodies. Indeed, from (19) it follows that $U_{(1)} \leq U_{(2)}$ where

$$
U_{(\alpha)}=\frac{1}{2} \lambda_{S}^{(\alpha)} \operatorname{tr}^{2} \epsilon+\mu_{S}^{(\alpha)} \epsilon: \boldsymbol{\epsilon}, \quad \alpha=1,2 .
$$

The corresponding energy spaces $\boldsymbol{E}_{(1)}$ and $\boldsymbol{E}_{(2)}$ for the problems coincide up to the form of the energy norms, which are equivalent, and so the infimum is taken over the same set of elements $\boldsymbol{E}_{(1)}=\boldsymbol{E}_{(2)}=\boldsymbol{E}$. Hence

$$
\left(\omega_{\min }^{(1)}\right)^{2}=\inf _{\boldsymbol{w} \in \boldsymbol{E}} R_{(1)}(\boldsymbol{w}) \leq \inf _{\boldsymbol{w} \in \boldsymbol{E}} R_{(2)}(\boldsymbol{w})=\left(\omega_{\min }^{(2)}\right)^{2} .
$$

Now we demonstrate that the least eigenfrequency depends continuously on the values of $\lambda_{S}$ and $\mu_{S}$.

Theorem 3.3. For any number $\varepsilon>0$, there exists a number $\delta>0$ such that $\left|\omega_{\min }^{(1)}-\omega_{\min }^{(2)}\right| \leq \varepsilon$ whenever $\left|\mu_{S}^{(1)}-\mu_{S}^{(2)}\right| \leq \delta$ and $\left|\lambda_{S}^{(1)}-\lambda_{S}^{(2)}\right| \leq \delta$.

Proof. We get

$$
\begin{aligned}
R_{(1)}(\boldsymbol{w})-R_{(2)}(\boldsymbol{w}) & =\frac{\int_{\Omega_{2}}\left[\left(\lambda_{S}^{(1)}-\lambda_{S}^{(2)}\right) \operatorname{tr}^{2} \boldsymbol{\epsilon}(\boldsymbol{w})+2\left(\mu_{S}^{(1)}-\mu_{S}^{(2)}\right) \boldsymbol{\epsilon}(\boldsymbol{w}): \boldsymbol{\epsilon}(\boldsymbol{w})\right] d \Omega}{K(\boldsymbol{w})} \\
& \leq \frac{\left|\lambda_{S}^{(1)}-\lambda_{S}^{(2)}\right| \int_{\Omega_{2}} \operatorname{tr}^{2} \boldsymbol{\epsilon}(\boldsymbol{w}) d \Omega+2\left|\mu_{S}^{(1)}-\mu_{S}^{(2)}\right| \int_{\Omega_{2}} \boldsymbol{\epsilon}(\boldsymbol{w}): \boldsymbol{\epsilon}(\boldsymbol{w}) d \Omega}{K(\boldsymbol{w})} \\
& \leq \delta \frac{\int_{\Omega_{2}}\left[\operatorname{tr}^{2} \boldsymbol{\epsilon}(\boldsymbol{w})+2 \boldsymbol{\epsilon}(\boldsymbol{w}): \boldsymbol{\epsilon}(\boldsymbol{w})\right] d \Omega}{K(\boldsymbol{w})}
\end{aligned}
$$

Taking the infimum over $\boldsymbol{E}$ of both sides of the inequality, we have

$$
\left(\omega_{\text {min }}^{(1)}\right)^{2}-\left(\omega_{\text {min }}^{(2)}\right)^{2} \leq \delta \inf _{\boldsymbol{w} \in \boldsymbol{E}} \frac{\int_{\Omega_{2}}\left[\operatorname{tr}^{2} \boldsymbol{\epsilon}(\boldsymbol{w})+2 \boldsymbol{\epsilon}(\boldsymbol{w}): \boldsymbol{\epsilon}(\boldsymbol{w})\right] d \Omega}{K(\boldsymbol{w})},
$$

which completes the proof.

From Theorem 3.3 we deduce an important

Corollary 3. The least eigenfrequency of a bounded elastic body with surface stresses tends to the least eigenfrequency for the same body with free boundary $\Omega_{2}$ :

$$
\omega_{\min } \rightarrow \omega_{\min }^{f} \quad \text { as } \quad \lambda_{S} \rightarrow 0 \text { and } \mu_{S} \rightarrow 0 .
$$

Proof. Starting with $R(\boldsymbol{w})-R_{0}(\boldsymbol{w})$ and repeating the transformations from the proof of Theorem 3.3, we obtain

$$
0 \leq R(\boldsymbol{w})-R_{0}(\boldsymbol{w}) \leq \delta \frac{\int_{\Omega_{2}}\left[\operatorname{tr}^{2} \boldsymbol{\epsilon}(\boldsymbol{w})+2 \boldsymbol{\epsilon}(\boldsymbol{w}): \boldsymbol{\epsilon}(\boldsymbol{w})\right] d \Omega}{K(\boldsymbol{w})}
$$

where $\delta=\max \left\{\left|\lambda_{S}\right|,\left|\mu_{S}\right|\right\}$. The set $\boldsymbol{E}$ is dense in $\boldsymbol{H}$, as $\boldsymbol{E}$ and $\boldsymbol{H}$ are the completions of the same space $\left(C^{(2)}(\bar{V})\right)^{3}$ with respect to different norms. Therefore

$$
\inf _{\boldsymbol{w} \in \boldsymbol{E}} R_{0}(\boldsymbol{w})=\inf _{\boldsymbol{w} \in \boldsymbol{H}} R_{0}(\boldsymbol{w})=\left(\omega_{\min }^{f}\right)^{2},
$$

and we get

$$
0 \leq\left(\omega_{\min }\right)^{2}-\left(\omega_{\min }^{f}\right)^{2} \leq \delta \inf _{\boldsymbol{w} \in \boldsymbol{E}} \frac{\int_{\Omega_{2}}\left[\operatorname{tr}^{2} \boldsymbol{\epsilon}(\boldsymbol{w})+2 \boldsymbol{\epsilon}(\boldsymbol{w}): \boldsymbol{\epsilon}(\boldsymbol{w})\right] d \Omega}{K(\boldsymbol{w})} \rightarrow 0 \quad \text { as } \quad \delta \rightarrow 0 .
$$

Note that Theorems 3.1-3.3 hold when $\lambda_{S}$ and $\mu_{S}$ are piecewise continuous on $\Omega_{2}$. In other words, they hold for a body with nonhomogeneous surface properties. 


\subsection{Higher eigenfrequencies}

The three eigenvalue Problems $P_{s s}, P_{f}$, and $P_{0}$ have discrete spectra, and the eigenmodes constitute complete orthogonal sets in the corresponding energy spaces and in the space $\left(L^{2}(V)\right)^{3}[2,14]$. The eigenfrequencies of these problems can be compared using Courant's minimax principle [5].

Courant's minimax principle. On one hand, Courant's principle allows us to find all the eigenfrequencies for each of the problems. For each problem we must repeat the proof of the statement on pp. 406-407 of [5], using an appropriate form of Rayleigh's quotient and an appropriate energy space. Our situation is even better than that presented in [5], however, as we have an existence theorem for the eigenmodes. In [5], existence was implicitly assumed.

On the other hand, Courant's principle allows us to compare the values of the eigenfrequencies for the problems under consideration. To this end we must reformulate the principle in such a way that the eigenfrequencies are determined using the same space, $\boldsymbol{H}$. The main point is that we should define the eigenfrequencies using a set of elements $\boldsymbol{H}$ that is common to all three problems under consideration. Moreover, for each problem we will use the same constraint $K(\boldsymbol{w})=1$. Under the imposed constraint, $R(\boldsymbol{w})=\|\boldsymbol{w}\|_{\boldsymbol{E}}^{2}$ and $R_{0}(\boldsymbol{w})=\|\boldsymbol{w}\|_{\boldsymbol{H}}^{2}$. Here the situation differs somewhat from that presented in [5]. As we have said, we extend the domain of $R$ by setting $R(\boldsymbol{w})=+\infty$ for the elements $\boldsymbol{w} \in \boldsymbol{H}$ that cannot be regarded as elements of $\boldsymbol{E}$.

Again, for the Problem $P_{s s}$ we can repeat the procedure stated on pp. 406-407 of [5], but on the space $\boldsymbol{H}$. This will yield the same set of eigenfrequencies that would be obtained from applying the procedure in $\boldsymbol{E}$.

The same is true for the eigenfrequencies of Problem $P_{0}$; we can define them using Courant's procedure in the space $\boldsymbol{H}$, while selecting from the orthogonal subspace $\boldsymbol{H}_{\perp}^{(k)}$ the elements that vanish on $\Omega$.

We now describe in detail the first step of Courant's principle, which allows us to compare the eigenvalues for the three problems.

1. Denote by $\boldsymbol{H}^{(k)}$ the subspace of $\boldsymbol{H}$ spanned by $k-1$ arbitrary chosen elements $\boldsymbol{v}_{1}, \boldsymbol{v}_{2}, \ldots, \boldsymbol{v}_{k-1}$ of $\boldsymbol{H}, k>1$. The space $\boldsymbol{H}_{\perp}^{(k)}$ is its "orthogonal" complement in $\boldsymbol{H}$ :

$$
\boldsymbol{H}_{\perp}^{(k)}=\left\{\boldsymbol{w} \in \boldsymbol{H} \mid\left\langle\boldsymbol{w}, \boldsymbol{v}_{1}\right\rangle_{\boldsymbol{L}}=\left\langle\boldsymbol{w}, \boldsymbol{v}_{2}\right\rangle_{\boldsymbol{L}}=\cdots=\left\langle\boldsymbol{w}, \boldsymbol{v}_{k-1}\right\rangle_{\boldsymbol{L}}=0\right\}
$$

where

$$
\langle\boldsymbol{u}, \boldsymbol{v}\rangle_{\boldsymbol{L}}=\int_{V} \rho \boldsymbol{u} \cdot \boldsymbol{v} d V
$$

$\boldsymbol{H}_{\perp}^{(k)}$ is a closed subspace of $\boldsymbol{H}$. By $\widehat{\boldsymbol{H}}_{\perp}^{(k)}$ we denote the subset of elements of $\boldsymbol{H}_{\perp}^{(k)}$ with the constraint $\langle\boldsymbol{w}, \boldsymbol{w}\rangle_{\boldsymbol{L}}=1$, i.e.,

$$
\widehat{\boldsymbol{H}}_{\perp}^{(k)}=\left\{\boldsymbol{w} \in \boldsymbol{H}_{\perp}^{(k)} \mid\langle\boldsymbol{w}, \boldsymbol{w}\rangle_{\boldsymbol{L}}=1\right\}
$$

2. Define

$$
\begin{aligned}
& d_{s s}\left[\boldsymbol{v}_{1}, \boldsymbol{v}_{2}, \ldots, \boldsymbol{v}_{k-1}\right]=\inf _{\widehat{\boldsymbol{H}}_{\perp}^{(k)} \cap \boldsymbol{E}}\|\boldsymbol{w}\|_{\boldsymbol{E}}^{2}, \\
& d_{f}\left[\boldsymbol{v}_{1}, \boldsymbol{v}_{2}, \ldots, \boldsymbol{v}_{k-1}\right]=\inf _{\widehat{\boldsymbol{H}}_{\perp}^{(k)}}\|\boldsymbol{w}\|_{\boldsymbol{H}}^{2}, \\
& d_{0}\left[\boldsymbol{v}_{1}, \boldsymbol{v}_{2}, \ldots, \boldsymbol{v}_{k-1}\right]=\inf _{\widehat{\boldsymbol{H}}_{\perp}^{(k)} \cap \boldsymbol{H}_{0}}\|\boldsymbol{w}\|_{\boldsymbol{H}}^{2} .
\end{aligned}
$$

3. Repetition of the proof of Courant's principle ( [5], pp. 406-407) shows that by taking the supremum of these quantities over all possible combinations $\boldsymbol{v}_{1}, \boldsymbol{v}_{2}, \ldots, \boldsymbol{v}_{k-1}$ in $\boldsymbol{H}$, we obtain the following eigenfrequencies.

For Problem $P_{\text {ss }}$ :

$$
\omega_{k}^{2}=\sup _{\boldsymbol{v}_{1}, \ldots, \boldsymbol{v}_{k-1}} d_{s s}\left[\boldsymbol{v}_{1}, \boldsymbol{v}_{2}, \ldots, \boldsymbol{v}_{k-1}\right]
$$


For Problem $P_{f}$ :

$$
\omega_{k}^{f^{2}}=\sup _{\boldsymbol{v}_{1}, \ldots, \boldsymbol{v}_{k-1}} d_{f}\left[\boldsymbol{v}_{1}, \boldsymbol{v}_{2}, \ldots, \boldsymbol{v}_{k-1}\right] .
$$

For Problem $P_{0}$ :

$$
\omega_{k}^{\circ 2}=\sup _{\boldsymbol{v}_{1}, \ldots, \boldsymbol{v}_{k-1}} d_{0}\left[\boldsymbol{v}_{1}, \boldsymbol{v}_{2}, \ldots, \boldsymbol{v}_{k-1}\right]
$$

These maximum-minimum values are attained if the elements $\boldsymbol{v}_{1}, \boldsymbol{v}_{2}, \ldots, \boldsymbol{v}_{k-1}$ coincide with the first $k-1$ eigenmodes of the corresponding eigenfrequency problem.

We will prove item 3 for Problem $P_{s s}$. Recall that the eigenvalues are ordered as $0<\omega_{\min }=\omega_{1} \leq \omega_{2}, \ldots$ and that to each $\omega_{k}$ there corresponds a unique eigenmode $\boldsymbol{w}_{k}$. Moreover, the relations (14) hold for the eigenmodes. First we note that $d_{s s}\left[\boldsymbol{w}_{1}, \boldsymbol{w}_{2}, \ldots, \boldsymbol{w}_{k-1}\right]=\omega_{k}^{2}$. Indeed, for elements $\boldsymbol{w} \in \widehat{\boldsymbol{H}}_{\perp}^{(k)}$ that do not belong to $\boldsymbol{E}$, we obtain $\|\boldsymbol{w}\|_{\boldsymbol{E}}^{2}=\infty$ so the infimum is sought on the set $\widehat{\boldsymbol{H}}_{\perp}^{(k)} \cap \boldsymbol{E}$. However, this is the procedure by which $\omega_{k}^{2}$ was found in [2].

Now we show that for any $\boldsymbol{v}_{1}, \boldsymbol{v}_{2}, \ldots, \boldsymbol{v}_{k-1} \in \boldsymbol{H}$ we have $d_{s s}\left[\boldsymbol{v}_{1}, \boldsymbol{v}_{2}, \ldots, \boldsymbol{v}_{k-1}\right] \leq \omega_{k}^{2}$. It suffices to find an element $\boldsymbol{v} \in \widehat{\boldsymbol{H}}_{\perp}^{(k)}$ for which $\|\boldsymbol{v}\|_{\boldsymbol{E}} \leq \omega_{k}^{2}$. Let us take a linear combination $\boldsymbol{v}=\sum_{m=1}^{k} c_{m} \boldsymbol{w}_{m}$, with constants $c_{1}, c_{2}, \ldots, c_{k}$, of the first $k$ eigenmodes $\boldsymbol{w}_{1}, \boldsymbol{w}_{2}, \ldots, \boldsymbol{w}_{k}$ that belongs to $\widehat{\boldsymbol{H}}_{\perp}^{(k)} \cap \boldsymbol{E}$. Such a combination exists because the elements $\boldsymbol{w}_{1}, \boldsymbol{w}_{2}, \ldots, \boldsymbol{w}_{k}$ are linearly independent while the dimension of $\boldsymbol{H}^{(k)}$ spanned by $\boldsymbol{v}_{1}, \boldsymbol{v}_{2}, \ldots, \boldsymbol{v}_{k-1} \in \boldsymbol{H}$ is no more than $k-1$. By (14) we obtain

$$
\begin{aligned}
\langle\boldsymbol{v}, \boldsymbol{v}\rangle_{\boldsymbol{L}} & =\left\langle\sum_{m=1}^{k} c_{m} \boldsymbol{w}_{m}, \sum_{n=1}^{k} c_{n} \boldsymbol{w}_{n}\right\rangle_{\boldsymbol{L}} \\
& =\sum_{m, n=1}^{k} c_{m} c_{n}\left\langle\boldsymbol{w}_{m}, \boldsymbol{w}_{n}\right\rangle_{\boldsymbol{L}} \\
& =\sum_{m=1}^{k} c_{m}^{2} .
\end{aligned}
$$

Since $\boldsymbol{v} \in \widehat{\boldsymbol{H}}_{\perp}^{(k)}$ is normalized with $\langle\boldsymbol{v}, \boldsymbol{v}\rangle_{\boldsymbol{L}}=1$, we obtain the normalization condition for the constants:

$$
\sum_{m=1}^{k} c_{m}^{2}=1 .
$$

Now we recall that $\left\langle\boldsymbol{w}_{m}, \boldsymbol{w}_{n}\right\rangle_{\boldsymbol{E}}=\omega_{n}^{2} \delta_{m n}$. It follows that

$$
\begin{aligned}
\|\boldsymbol{v}\|_{\boldsymbol{E}}^{2} & =\left\langle\sum_{m=1}^{k} c_{m} \boldsymbol{w}_{m}, \sum_{n=1}^{k} c_{n} \boldsymbol{w}_{n}\right\rangle_{\boldsymbol{E}} \\
& =\sum_{m, n=1}^{k} c_{m} c_{n}\left\langle\boldsymbol{w}_{m}, \boldsymbol{w}_{n}\right\rangle_{\boldsymbol{E}} \\
& =\sum_{m, n=1}^{k} c_{m} c_{n} \omega_{n}^{2} \delta_{m n} \\
& =\sum_{m=1}^{k} c_{m}^{2} \omega_{m}^{2} \\
& \leq \sum_{m=1}^{k} c_{m}^{2} \omega_{k}^{2} \\
& =\omega_{k}^{2},
\end{aligned}
$$

which completes the proof. 
In contrast to [5], we have not implicitly assumed the existence of the eigenmodes for the corresponding boundary value problems.

A process of comparison permits us to extend the inequalities (18) and (20) to higher eigenfrequencies. This is addressed in

Theorem 3.4. Let $\omega_{k}$ be eigenfrequencies of a bounded elastic body with surface stresses enumerated in increasing order as $\omega_{0} \leq \omega_{1} \leq \omega_{2}, \ldots$, and let $\omega_{k}^{f}$ and $\omega_{k}^{\circ}$ be correspondingly ordered eigenfrequencies of the elastic body with free boundary $\Omega_{2}$ and with fixed boundary, respectively. Then

$$
\omega_{k}^{f} \leq \omega_{k} \leq \omega_{k}^{\circ}, \quad k=1,2,3, \ldots
$$

Proof. First we prove the left-hand inequality of (21). Since $\|\boldsymbol{w}\|_{\boldsymbol{H}} \leq\|\boldsymbol{w}\|_{\boldsymbol{E}}$, we have

$$
d_{f}\left[\boldsymbol{v}_{1}, \boldsymbol{v}_{2}, \ldots, \boldsymbol{v}_{k-1}\right] \leq \inf _{\widehat{H}_{\perp}^{(k)}}\|\boldsymbol{w}\|_{\boldsymbol{E}}^{2} \leq d_{s s}\left[\boldsymbol{v}_{1}, \boldsymbol{v}_{2}, \ldots, \boldsymbol{v}_{k-1}\right]
$$

Thus, for the greatest values of $d_{f}$ and $d_{s s}$ we obtain

$$
\sup _{\boldsymbol{v}_{1}, \ldots, \boldsymbol{v}_{k-1}} d_{f}\left[\boldsymbol{v}_{1}, \boldsymbol{v}_{2}, \ldots, \boldsymbol{v}_{k-1}\right] \leq \sup _{\boldsymbol{v}_{1}, \ldots, \boldsymbol{v}_{k-1}} d_{s s}\left[\boldsymbol{v}_{1}, \boldsymbol{v}_{2}, \ldots, \boldsymbol{v}_{k-1}\right]
$$

We conclude that $\omega_{k}^{f^{2}} \leq \omega_{k}^{2}$. The right-hand inequality of (21) is proved in a similar fashion. When $\boldsymbol{w} \in \boldsymbol{H}_{0}$, we see that $\|\boldsymbol{w}\|_{\boldsymbol{E}}=\|\boldsymbol{w}\|_{\boldsymbol{H}}$. So we have

$$
d_{0}\left[\boldsymbol{v}_{1}, \boldsymbol{v}_{2}, \ldots, \boldsymbol{v}_{k-1}\right]=\inf _{\widehat{\boldsymbol{H}}_{\perp}^{(k)} \cap \boldsymbol{H}_{0}}\|\boldsymbol{w}\|_{\boldsymbol{H}}^{2}=\inf _{\widehat{\boldsymbol{H}}_{\perp}^{(k)} \cap \boldsymbol{H}_{0}}\|\boldsymbol{w}\|_{\boldsymbol{E}}^{2}
$$

But

$$
\inf _{\widehat{H}_{\perp}^{(k)} \cap \boldsymbol{H}_{0}}\|\boldsymbol{w}\|_{\boldsymbol{E}}^{2} \geq \inf _{\widehat{\boldsymbol{H}}_{\perp}^{(k)}}\|\boldsymbol{w}\|_{\boldsymbol{E}}^{2}
$$

So

$$
d_{0}\left[\boldsymbol{v}_{1}, \boldsymbol{v}_{2}, \ldots, \boldsymbol{v}_{k-1}\right] \geq d_{s s}\left[\boldsymbol{v}_{1}, \boldsymbol{v}_{2}, \ldots, \boldsymbol{v}_{k-1}\right]
$$

Thus

$$
\omega_{k}^{2}=\sup _{\boldsymbol{v}_{1}, \ldots, \boldsymbol{v}_{k-1}} d_{s s}\left[\boldsymbol{v}_{1}, \boldsymbol{v}_{2}, \ldots, \boldsymbol{v}_{k-1}\right] \leq \sup _{\boldsymbol{v}_{1}, \ldots, \boldsymbol{v}_{k-1}} d_{0}\left[\boldsymbol{v}_{1}, \boldsymbol{v}_{2}, \ldots, \boldsymbol{v}_{k-1}\right]=\omega_{k}^{\circ 2}
$$

In a similar way, using Courant's principle, inequality (20) can be extended to higher eigenfrequencies.

Theorem 3.5. Let $\omega_{k}^{(1)}$ be eigenfrequencies of a bounded elastic body with moduli $\lambda, \mu$ and surface elastic moduli $\lambda_{S}^{(1)}, \mu_{S}^{(1)}$, ordered as $\omega_{0}^{(1)} \leq \omega_{1}^{(1)} \leq \omega_{2}^{(1)}, \ldots$. Let $\omega_{k}^{(2)}$ be the ordered eigenfrequencies for the elastic body with moduli $\lambda, \mu$ but with surface moduli $\lambda_{S}^{(2)}, \mu_{S}^{(2)}$. Let

$$
\mu_{S}^{(1)} \leq \mu_{S}^{(2)}, \quad \lambda_{S}^{(1)}+\mu_{S}^{(1)} \leq \lambda_{S}^{(2)}+\mu_{S}^{(2)} .
$$

Then

$$
\omega_{k}^{(1)} \leq \omega_{k}^{(2)} \quad \text { for } \quad k=1,2,3, \ldots
$$

Proof. For the body with surface elastic moduli $\lambda_{S}^{(1)}, \mu_{S}^{(1)}$ we denote the energy space by $\boldsymbol{E}_{(1)}$ and assign the superscript (1) to all corresponding quantities. Similarly, $\boldsymbol{E}_{(2)}$ denotes the energy space for the body with surface elastic moduli and $\lambda_{S}^{(2)}, \mu_{S}^{(2)}$. The $k$ th eigenfrequency of the $\alpha$ th body with surface stresses is given by

$$
\left(\omega_{k}^{(\alpha)}\right)^{2}=\sup _{\boldsymbol{v}_{1}, \ldots, \boldsymbol{v}_{k-1}} \widehat{\inf }_{\perp}^{(k)}\|\boldsymbol{w}\|_{\boldsymbol{E}_{(\alpha)}^{2}}^{2}, \quad \alpha=1,2 .
$$


Although the expressions for the norms of $\boldsymbol{E}_{(1)}$ and $\boldsymbol{E}_{(2)}$ are distinct, the spaces contain the same elements. Moreover, $\|\boldsymbol{w}\|_{\boldsymbol{E}_{(1)}} \leq\|\boldsymbol{w}\|_{\boldsymbol{E}_{(2)}}$. For the supremum we obtain

$$
\sup _{\boldsymbol{v}_{1}, \ldots, \boldsymbol{v}_{k-1}} \inf _{\widehat{\boldsymbol{H}}_{\perp}^{(k)}}\|\boldsymbol{w}\|_{\boldsymbol{E}_{(1)}}^{2} \leq \sup _{\boldsymbol{v}_{1}, \ldots, \boldsymbol{v}_{k-1}} \inf _{\widehat{\boldsymbol{H}}_{\perp}^{(k)}}\|\boldsymbol{w}\|_{\boldsymbol{E}_{(2)}^{2}}
$$

which completes the proof.

Theorems 3.1-3.5 can be proved for more general boundary conditions on $\Omega_{1}$. For example, they hold true for boundary conditions of the form

$$
\left.\boldsymbol{w}\right|_{\Omega_{1}^{(1)}}=\mathbf{0},\left.\quad \boldsymbol{n} \cdot \boldsymbol{\sigma}\right|_{\Omega_{1}^{(2)}}=\mathbf{0},\left.\quad \boldsymbol{n} \cdot \boldsymbol{w}\right|_{\Omega_{1}^{(3)}}=0,\left.\quad \boldsymbol{n} \cdot \boldsymbol{\sigma} \cdot(\mathbf{I}-\boldsymbol{n} \otimes \boldsymbol{n})\right|_{\Omega_{1}^{(3)}}=\mathbf{0},
$$

where $\Omega_{1}=\Omega_{1}^{(1)} \cup \Omega_{1}^{(2)} \cup \Omega_{1}^{(3)}, \Omega_{1}^{(1)} \cap \Omega_{1}^{(2)}=\varnothing$, and $\Omega_{1}^{(2)} \cap \Omega_{1}^{(3)}=\varnothing$.

These theorems can also be proved when $\Omega_{1}=\varnothing$, i.e., when Problems $P_{f}$ and $P_{s s}$ are formulated for a body free from geometrical constraints. In this case the first six eigenfrequencies are equal to zero, and the corresponding eigenmodes constitute the basis for the translations and rotations of a rigid body.

\section{Example: radial oscillations of an elastic sphere with surface stresses}

To illustrate the spectral properties of Problems $P_{s s}, P_{f}$, and $P_{0}$, we consider the oscillations of an elastic sphere - a problem that admits analytical solution. The radial eigen-vibrations of an elastic sphere are treated in many textbooks, e.g., $[16,17]$. For the radial vibrations, the displacement field is

$$
\boldsymbol{w}=w(r) \boldsymbol{e}_{r}
$$

where $r$ and $\boldsymbol{e}_{r}$ are the radius and the corresponding basis vector of spherical coordinates. By spherical symmetry it follows that

$$
w(0)=0 .
$$

For the radially symmetric problem, the point $r=0$ corresponds to $\Omega_{1}$. Substituting $w(r)=r f(r)$ into $(10)_{1}$, we reduce the equation to $[16,17]$

$$
f^{\prime \prime}+\frac{4}{r} f^{\prime}+\eta^{2} f=0, \quad \text { where } \quad \eta^{2}=\frac{\rho \omega^{2}}{\lambda+2 \mu} .
$$

The solution of (25) satisfying (24) is

$$
f(r)=\frac{\eta r \cos \eta r-\sin \eta r}{r^{3}} .
$$

Then the radial component of the stress tensor $\sigma$ is

$$
\sigma_{r}(r)=(2 \mu+\lambda) w^{\prime}(r)+2 \lambda \frac{w(r)}{r} .
$$

Let us consider the surface stresses in the case of radial deformation. The boundary portion $\Omega_{2}$ is $r=a$, where $a$ is the radius of the ball. In spherical coordinates,

$$
\nabla_{S}=\frac{1}{a}\left(e_{\theta} \frac{\partial}{\partial \theta}+e_{\phi} \frac{1}{\sin \theta} \frac{\partial}{\partial \phi}\right)
$$

where $\phi, \theta$ are the angular coordinate variables and $\boldsymbol{e}_{\theta}, \boldsymbol{e}_{\phi}$ are their corresponding basis vectors [13]. So

$$
\boldsymbol{\epsilon}=\frac{w(a)}{a}\left(\boldsymbol{e}_{\theta} \boldsymbol{e}_{\theta}+\boldsymbol{e}_{\phi} \boldsymbol{e}_{\phi}\right) \equiv \frac{w(a)}{a} \mathbf{A} .
$$

Substituting this into (6), we obtain the surface stress tensor and its divergence:

$$
\boldsymbol{\tau}=\tau \mathbf{A}, \quad \tau=2\left(\mu_{S}+\lambda_{S}\right) \frac{w(a)}{a}, \quad \nabla_{S} \cdot \boldsymbol{\tau}=-\frac{2 \tau}{a} \boldsymbol{n} .
$$


The boundary conditions for the fixed boundary, the free boundary, and the boundary with surface stresses, i.e., for Problems $P_{0}, P_{f}$, and $P_{s s}$, reduce to

$$
\begin{aligned}
& w(a)=0, \\
& \sigma_{r}(a)=0, \\
& \sigma_{r}(a)=-\frac{2 \tau}{a},
\end{aligned}
$$

respectively. Using (26), we transform Eqs. (27)-(29) to the following transcendental equations in $\eta$ :

$$
\begin{aligned}
& \eta a \cos (\eta a)-\sin (\eta a)=0, \\
& (\lambda+2 \mu) \eta^{2} a^{2} \sin (\eta a)+4 \mu[\eta a \cos (\eta a)-\sin (\eta a)]=0, \\
& (\lambda+2 \mu) \eta^{2} a^{2} \sin (\eta a)+4 \mu[\eta a \cos (\eta a)-\sin (\eta a)]-\alpha(\cos (\eta a) \eta a-\sin (\eta a))=0,
\end{aligned}
$$

where $\alpha=4\left(\lambda_{S}+\mu_{S}\right) / \mu a$ is a dimensionless parameter. We denote the solutions of (30)-(32) by $\eta_{k}^{\circ}$, $\eta_{k}^{f}$, and $\eta_{k}$, respectively. Since $\alpha$ in (32) depends on $a$ explicitly, the solutions of (32) depend on the radius of the ball. Note that $\alpha \rightarrow 0$ as $a \rightarrow \infty$, while $\alpha \rightarrow \infty$ as $a \rightarrow 0$. With the given elastic moduli, the eigenfrequency depends on $a$, which is a so-called size effect. It is obvious that (32) reduces to (30) when $\alpha=0$ and to (31) when $\alpha \rightarrow \infty$. It follows that $\eta_{k}=\eta_{k}^{f}$ when $\alpha=0$ and $\eta_{k} \rightarrow \eta_{k}^{\circ}$ when $\alpha \rightarrow \infty$. So the bounds (21) cannot be strengthened, in general.

Table 1 Normalized eigenfrequencies of an elastic sphere for Problems $P_{f}, P_{s s}$, and $P_{0}$.

\begin{tabular}{c|c|c|c|c|c|c|c}
\hline$k$ & 1 & 2 & 3 & 4 & 5 & 6 & 7 \\
\hline$\eta_{k}^{f} / a$ & 2.563434163 & 6.058670084 & 9.279861114 & 12.45884069 & 15.62235640 & 18.77840320 & 21.93025612 \\
$\eta_{k} / a$ & 2.743707270 & 6.116764264 & 9.316615629 & 12.48593737 & 15.64386611 & 18.79625335 & 21.94551807 \\
$\eta_{k}^{\circ} / a$ & 4.493409458 & 7.725251837 & 10.90412166 & 14.06619391 & 17.22075527 & 20.37130296 & 23.51945250 \\
\hline
\end{tabular}

The first seven eigenfrequencies are presented in Table 1, assuming $\lambda=\mu$ and $\alpha=1$. For Problem $P_{s s}$, the dependencies of $\eta_{k}$ on $\alpha$ are given in Fig. 1. Here the dashed and stroke-dashed lines correspond to $\eta_{k}^{f}$ and $\eta_{k}^{\circ}$ for Problems $P_{f}$ and $P_{0}$, respectively.
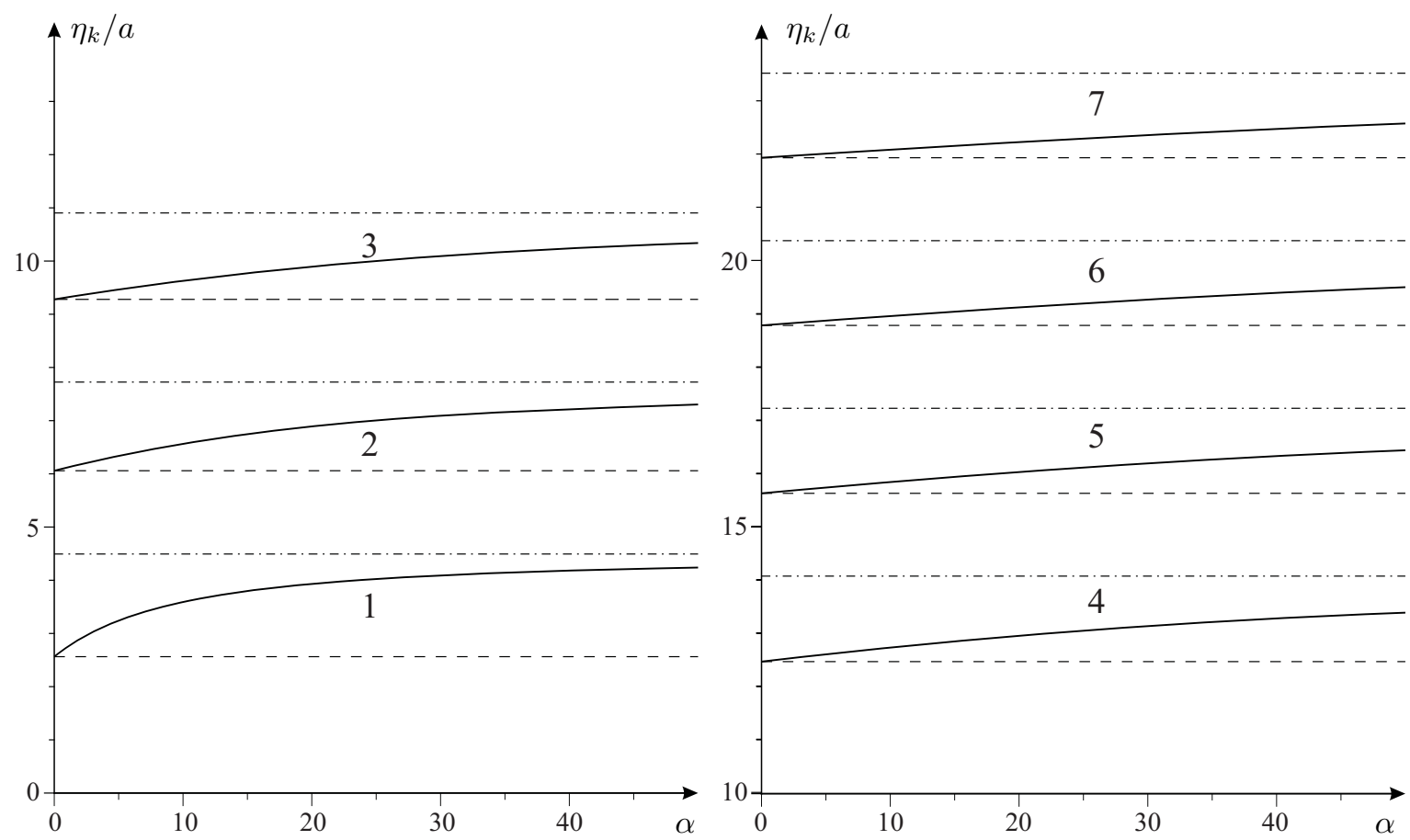

Fig. 1 Dependencies of the normalized eigenfrequencies $\eta_{k}$ on $\alpha$ for $k=1,2, \ldots, 7$. 


\section{Conclusions}

We established lower and upper bounds for the eigenfrequencies of an elastic body with surface stresses. These bounds cannot be improved. For the $k$ th eigenfrequency, the lower bound is the $k$ th eigenfrequency of the same body with free boundary, while the upper bound is the $k$ th eigenfrequency of the same body with fixed boundary (the eigenfrequencies are numbered in increasing order, taking into account multiplicity of the modes). An increase in the values of surface elastic moduli implies an increase in the eigenfrequencies. The proof is based on Rayleigh's minimal principle and Courant's maximum-minimum principle. The classical formulation of Courant's principle [5] is modified to account for the peculiarities of the eigenvalue problems under consideration.

The increase in the eigenfrequencies for the elastic body with surface stresses, in comparison with the same body with free boundary, can be interpreted as the increase in the stiffness. This conclusion coincides qualitatively with the results in $[6,7]$ for nano-porous or nano-cellular media, and with the increase in stiffness parameters of nanosized plates and shells $[3,8]$. The influence of the surface elasticity is more significant for higher eigenfrequencies and for bodies with surface imperfections. The stiffening effect is also more significant when the body sizes decrease, i.e., for nano-sized bodies. Note that for micro- and nano-sized solids, the initial (residual) surface stresses can significantly affect the apparent material properties [25, 27-29]. Residual surface stresses can increase or decrease the solid eigen-frequencies. From a mathematical viewpoint, the corresponding boundary-value problems are quite different from those we have considered; they are left as future work.

The general theory is illustrated by the free radial vibrations of an elastic sphere with surface stresses. For the vibrating sphere, the eigenfrequencies are obtained numerically and the influence of the surface elasticity properties is analyzed.

Acknowledgements The authors are grateful to M. J. Cloud of Lawrence Technological University, USA, for assistance in preparing this paper. The second author was supported by DFG grant No. AL 341/33-1 and by the RFBR under grant No. 09-01-00459.

\section{References}

[1] R. A. Adams and J. J. F. Fournier, Sobolev Spaces, 2nd edition, Pure and Applied Mathematics, Vol. 140 (Academic Press, Amsterdam, 2003).

[2] H. Altenbach, V. A. Eremeyev, and L. P. Lebedev, On the existence of solution in the linear elasticity with surface stresses, Z. Angew. Math. Mech. 90(7), 535-536 (2010).

[3] H. Altenbach, V. A. Eremeyev, and N. F. Morozov, Linear theory of shells taking into account surface stresses, Dokl. Phys. 54(12), 531-535 (2009).

[4] P. Ciarlet, Mathematical Elasticity. Vol. I: Three-Dimensional Elasticity (North-Holland, Amsterdam, 1988).

[5] R. Courant and D. Hilbert, Methods of Mathematical Physics. Vol. I (Wiley, Singapore, 1989).

[6] H. L. Duan, J. Wang, and B.L. Karihaloo, Theory of elasticity at the nanoscale, in: Advances in Applied Mechanics (Elsevier, 2008) pp. 1-68.

[7] H. L. Duan, J. Wang, B. L. Karihaloo, and Z.P. Huang, Nanoporous materials can be made stiffer than non-porous counterparts by surface modification, Acta Mater. 54, 2983-2990 (2006).

[8] V. A. Eremeyev, H. Altenbach, and N.F. Morozov, The influence of surface tension on the effective stiffness of nanosize plates, Dokl. Phys. 54(2), 98-100 (2009).

[9] G. Fichera, Existence theorems in elasticity, in: Handbuch der Physik, edited by S. Flügge (Springer, Berlin, 1972) pp. $347-389$.

[10] M.E. Gurtin, X. Markenscoff, and R. N. Thurston, Effect of surface stress on natural frequency of thin crystals, Appl. Phys. Lett. 29(9), 529-530 (1976).

[11] M.E. Gurtin and A. I. Murdoch, A continuum theory of elastic material surfaces, Arch. Ration. Mech. Anal. 57(4), 291-323 (1975).

[12] J. Lagowski, H. C. Gatos, and E. S. Sproles, Surface stress and normal mode of vibration of thin crystals: GaAs, Appl. Phys. Lett. 26(9), 493-495 (1975).

[13] L.P. Lebedev, M. J. Cloud, and V. A. Eremeyev, Tensor Analysis with Applications in Mechanics (World Scientific, New Jersey, 2010).

[14] L. P. Lebedev and I. I. Vorovich, Functional Analysis in Mechanics (Springer, New York, 2003).

[15] L. P. Lebedev, I. I. Vorovich, and G. M. L. Gladwell, Functional Analysis. Applications in Mechanics and Inverse Problems, 2nd edition, Solid Mechanics and Its Applications, Vol. 100 (Kluwer, New York, 2002).

[16] A. E. H. Love, A Treatise on the Mathematical Theory of Elasticity, 4th edition (Dover, New York, 1944).

[17] W. Nowacki, Theory of Elasticity (in Polish) (Państwowe Wydawnictwo Naukowe, Warsaw, 1970).

[18] Y.S. Podstrigach and Y.Z. Povstenko, Introduction to Mechanics of Surface Phenomena in Deformable Solids (in Russian) (Naukova Dumka, Kiev, 1985).

[19] Y.Z. Povstenko, Theoretical investigation of phenomena caused by heterogeneous surface tension in solids, J. Mech. Phys. Solids 41(9), 1499-1514 (1993).

[20] P. Schiavone and C. Q. Ru, Integral equation methods in plane-strain elasticity with boundary reinforcement, Proc. R. Soc. Lond. A, Math. Phys. Eng. Sci. 454(1976), 2223-2242 (1998). 
[21] P. Schiavone and C. Q. Ru, Solvability of boundary value problems in a theory of plane-strain elasticity with boundary reinforcement, Int. J. Eng. Sci. 47(11-12), 1331-1338 (2009).

[22] F. Song and G. L. Huang, Modeling of surface stress effects on bending behavior of nanowires: Incremental deformation theory, Phys. Lett. A 373(43), 3969-3973 (2009).

[23] D. J. Steigmann and R. W. Ogden, Elastic surface-substrate interactions, Proc. R. Soc. Lond. A, Math. Phys. Eng. Sci. 455(1982), 437-474 (1999).

[24] S. Timoshenko, Vibration Problems in Engineering, 2nd edition (D. van Nostrand Company, New York, 1928); W. Weaver, S. Timoshenko, and D. H. Young, Vibration Problems in Engineering, 5th edition (Wiley, New York, 1990).

[25] G. F. Wang and X. Q. Feng, Effects of surface elasticity and residual surface tension on the natural frequency of microbeams, Appl. Phys. Lett. 90(23), 231904 (2007).

[26] G. F. Wang and X. Q. Feng, Effect of surface stresses on the vibration and buckling of piezoelectric nanowires, Europhys. Lett. 91(5), 56007 (2010).

[27] Z. Q. Wang, Y. P. Zhao, and Z.P. Huang, The effects of surface tension on the elastic properties of nano structures, Int. J. Eng. Sci. 48(2), 140-150 (2010).

[28] H.X. Zhu, The effects of surface and initial stresses on the bending stiffness of nanowires, Nanotechnology 19(40), 405703 (2008).

[29] H. X. Zhu, J.X. Wang, and B. L. Karihaloo, Effects of surface and initial stresses on the bending stiffness of trilayer plates and nanofilms, J. Mech. Mater. Struct. 4(3), 589-604 (2009). 